\title{
Full Probabilistic Characteristics of Power Losses in the Electrical Power System Branches
}

\author{
Yuly BAY ${ }^{1}$, Igor RAZZHIVIN ${ }^{1}$, Anton KIEVETS ${ }^{1}$, Mikhail ANDREEV ${ }^{1}$, Vladimir RUDNIK ${ }^{1}$ \\ ${ }^{1}$ Division for Power and Electrical Engineering, School of Energy and Power Engineering, Tomsk Polytechnic \\ University, Russia
}

\begin{abstract}
Stable operation of the electrical power system (EPS) is one of the main issues considered in the power industry. Current levels of electricity consumption lead to the need to increase the generated capacity, repeatedly converting and complicating the original circuit. In addition to this, given the current trend towards the use of renewable energy sources (RES), more and more uncertainties are added, that are difficult to predict. Events in the EPS, and especially in the case of RES, are deterministic, i.e. random. This leads to the fact that it is difficult to fully assess the EPS stability and the possible power loss. It is also difficult to determine the amount of permissible power generated by RES, which will not lead to subsequent mode violations. The purpose of this article is to test the developed SIBD method for obtaining the full probabilistic characteristics of power losses in each branch. This method, unlike the Monte Carlo methods, does not use a random sample of initial data, but completely covers the studied functional dependence (FD). The method is used to obtain the probability distribution laws (PDLs) of power losses in transmission lines based on unmodified IEEE 30-Bus and IEEE 14-Bus systems and their examination. These laws are necessary for further determination of the optimal EPS operating modes, to solve the problem of determining the optimal RES installation, the required amount of renewable generated energy in a nondeterministic way.
\end{abstract}

Keywords: probability density function, random variable, quantity, electric power system, power losses.

Received: 13 May 2020

To cite this article:

BAY Y., RAZZHIVIN I., KIEVETS A., ANDREEV M., RUDNIK V., "Obtaining Full Probabilistic Characteristics of Power Losses in the Electrical Power System Branches" in Electrotehnica, Electronica, Automatica (EEA), 2020, vol. 68, no. 3, pp. 32-40, ISSN 1582-5175. https://doi.org/10.46904/eea.20.68.3.1108004

\section{Introduction}

During recent years, ensuring the EPS secure planning has become an important issue along with its development. There is currently a great demand for electricity all over the world, but production is not enough to meet demand. Due to this, the penetration of RES into the EPS has expanded. In future, a large share of the energy [1] will be generated with wind power plants (WPPs) and other RES, such as solar power cells. As a result, more and more uncertainties are introduced which makes power system uncontrolled.

This is due to the fact, that the system normally has a classical "vertically-operated" power system there is only a "small" number of large centralized generators dispatchable, i.e. controllable to meet the demand. However, in the "horizontally-operated" power system, the DG units in the "active" distribution networks are practically non-dispatchable [2].

The Distribution network operators (DNO'S) are liable for retaining the reliability and efficiency of EPS. Thus, for optimal energy systems operation with minimal power losses and fluctuations, as well as the ability to install RES, it is necessary to determine a strict and adequate method to eliminate the uncertainties associated with these problems.
To find the magnitude of change in power loss, it is necessary to determine all possible losses that can flow through the transmission line of interest to research, as well as the total losses throughout the system. Therefore, it is necessary to determine in a nondeterministic form [3] how the power loss is distributed over the network, in order to their possible connection with installations for the active generation of electricity from RES, not in random (deterministic cases), but in a whole set of states.

The main problem is that obtaining the probabilistic characteristics by standard statistical methods has no full solution [4]. It is necessary to make a full assessment of the probabilistic characteristics of the EPS, including power losses, in order to understand the entire range of processes in the network. In this regard, the method of selection of interval boundaries of input and output data (SIBD) is proposed, and its application for obtaining probabilistic characteristics of electrical quantities and their imbalances.

\section{The Literature Review}

Obtaining the cumulative distribution function (CDF) and probability density function (PDF) of multidimensional FD, as well as probabilities of a FD assuming values from the multidimensional domain, in its 
canonical analytical variant represents a functional of a multidimensional PD within the limits determined by the essence of the problem.

There is the practical problem of the mass peculiarities of FDs that shape the boundaries of the area of the functional [5], which condition the immeasurable analytical complications of controlling the expressions of limits and the correct sequence of the algorithm for calculating the functional of the problem. In the electric power industry, these are first of all the multidimensional problems of determining the probabilistic characteristics. In the case of digital substations (DS), where a high level of automation [6], information exchange between DS elements through various protocols, the ability to predetermine the imbalances and manage them will affect the reliability and performance [7].

However, analytical difficulties of forming limits and analytical functionals calculation may arise starting from the third or the fourth FD dimension. It becomes pertinent to search for other methods of calculation that are less dependent on FD dimension.

Therefore, various methods associated with the numerical obtaining of probability characteristics are developed and used. For example, the calculation of FD values for each statistically modelled variant of a random variable (RV), which is used only to obtain statistics of FD, i.e. the requirements for the formation and calculation of functionals are not set at all, and the resulting statistics are used for their intended purpose to obtain statistical estimates of the probabilistic characteristics. Also, methods based on the expansion of the functional dependence with respect to the mathematical expectations of random arguments of the FD.

To preserve the statistical representativeness of the obtained results of multivariate $\mathrm{FD}$, the number of required test values of FD statistical estimates increases, which limits the use of methods in the case of FD for many RV. Based on this, it is relevant to search for other computational numerical methods for determining the PDL, not critically dependent on the dimension of the FD.

The most famous methods used in this field include the Monte Carlo methods [8] based on the creation of statistical data with the condition of statistical representativeness of the final result. The following methods also may be noted: the unsymmetrical two point estimate method (US2PEM) [9], the symmetrical two point estimate method (S2PEM) [10], The GramCharlier method, and Latin Hypercube Sampling (LHS), designed to replace the Monte Carlo method in a number of cases, allowable increase in error relative to Monte Carlo [11]. Also, can be note methods for finding the optimal state of the system, such as the Artificial Bee Colony and Genetic Algorithm, however, due to their deterministic logic, they are not suitable for this particular issue.

The presence of these methods does not implore the need to search for new ones that are able to fully investigate PDF uniformly, over the full range of functional dependence, without losing the "weight" of the partial density of a specific FD value.
In this regard, all complications in EPS lead to an increase in uncertainties, all further work should be aimed at their full assessment, since you need to be sure that the influence of stochastic objects in the system will not entail a violation of stability.

\section{Scientific Novelty}

In the FDs that describe practical problems, it is rational to consider all RVs as independent variables and, consequently, to change their values arbitrarily, irrespectively of the types of conditional probabilistic characteristics that change with the change of RV values, which is a natural advantage. In the case with dependent RVs, values of one RV must be determined using expressions through products of conditional probabilities, which represents additional counterproductive procedures that can always be avoided using high-quality logical selection of independent RVs or high-quality orthogonalization of the source dependent RVs.

The method of determining CDFs and PDFs of a FD, as well as that of the probability of an FD assuming a value from the interval of the CDF and PDF of random variables, consists in natural summation of probabilities of each variant of RV values combination according to different criteria. The manifold of RV value variants that corresponds to the FD value can be called an association of this value of the FD. Value-association of the FD that as an event consists of the total sum of variants of combining value-events of all RVs. Variants of combinations of these value-events are incompatible, because they are formed from all RVs and can't combine other than as different values of at least one RV. Values of each RV are incompatible relative to each other, so all variants of combining all RVs will be incompatible as well. Therefore, the probability of association-value of an FD equals the sum of probabilities of all RV combination-events that are associated with this value of the FD, and this probability can be called the eigenprobability of the FD value. More details about the method can be found here [12].

A method of selection of interval boundaries of input and output data (SIBD) that is not critical to FD dimension was proposed. The SIBD algorithm (Figure 1) is described by mathematical operations for explicit expressions for the PD. The PDFs for $X_{1}, \ldots X_{2}, \ldots X_{n} \mathrm{RV}$ are assumed to be known.

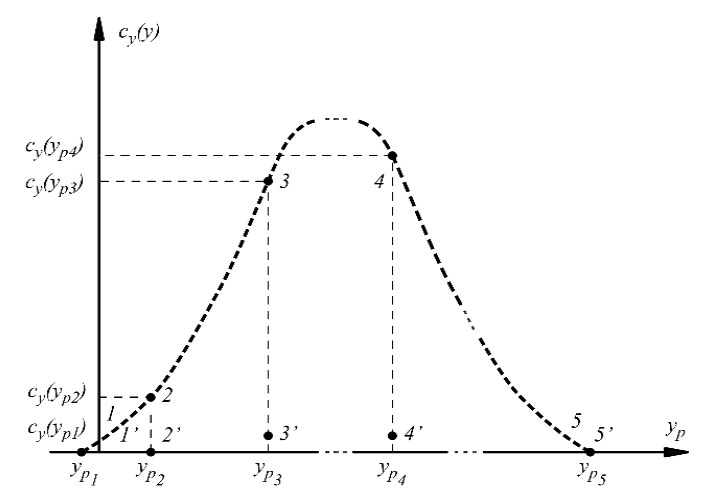

Figure 1. PDs of the sum of two independent uniform RVs 
1. Choosing the number and values of the PDF quantile orders: $0, p_{1}, \ldots p_{j}, \ldots 1$.

2. Calculating the PD values as quantiles of the same order. As example, quantile $\# 0$ is explained by the formula (1):

$$
\left\{\begin{array}{c}
x_{10}=\mathrm{f}_{1}^{-1}(0), \ldots, x_{i 0}=\mathrm{f}_{i}^{-1}(0), \ldots, x_{n 0}=\mathrm{f}_{n}^{-1}(0) \\
c_{1}\left(x_{10}\right), \ldots, c_{i}\left(x_{i 0}\right), \ldots, c_{n}\left(x_{n 0}\right)
\end{array}\right.
$$

Similarly, for other quantile orders $p_{1}, \ldots p_{j}, \ldots 1$.

3. Forming the FD main orders and compatible PD of the same order.

a) 0 order (2):

$$
\left\{\begin{array}{c}
y_{0}=\varphi\left(x_{10}, \ldots x_{i 0}, \ldots x_{n 0}\right) \\
c_{0}\left(x_{10}, \ldots x_{i 0}, \ldots x_{n 0}\right)=c_{1}\left(x_{10}\right) \ldots c_{i}\left(x_{i 0}\right) \ldots c_{n}\left(x_{n 0}\right)
\end{array}\right.
$$

b) $p_{1}$ order (3):

$$
\left\{\begin{array}{c}
y_{p_{1}}=\varphi\left(x_{1 p_{1}}, \ldots x_{i p_{1}}, \ldots x_{n p_{1}}\right) \\
c_{p_{1}}\left(x_{1 p_{1}}, \ldots x_{i p_{1}}, \ldots x_{n p_{1}}\right)=c_{1}\left(x_{1 p_{1}}\right) \ldots c_{i}\left(x_{i p_{1}}\right) \ldots c_{n}\left(x_{n p_{1}}\right)
\end{array}\right.
$$

Similarly, for other quantile orders .... $p_{j}, \ldots 1$.

4. Forming and exhaustive search the RV values as quantiles of the selected orders $X_{1}, \ldots X_{2}, \ldots X_{n}$ of FD $Y=\varphi\left(X_{1}, \ldots X_{2}, \ldots X_{n}\right)$, calculation of the FD values, compatible PD of RV, it selection and summing as a PD components according to the principle of equality to main orders FD.

\section{Method Description}

The deterministic problems solution of calculating the parameters of the modes is predetermined by the logic of the laws and methods of electrical engineering [13], [14]. The natural deterministic logic of the calculation procedure as applied to the formation of PDL values for damage is very costly.

This is because the mode parameters after the processing of the independent RV capacities of the load and generator nodes into the mode parameters FDs become statistically dependent on each other [15]. Obtaining conditional PDL mode parameters is possible, however, it will require an additional amount of computation, therefore, algorithms for determining the PDL should be single-stage and proceed from the same independent random arguments.

There can be a probabilistic dependence between the active and reactive power of the load nodes, the active power and voltage of the generator nodes of EPS. Since the components of these nodes are usually distributed over the normal PDL, the joint and conditional PDL will be normal, and the parameters of the latter are expressed through the correlation coefficient [16].

Intra-site dependencies between the values do not affect the dependence of the different node values. There is still no dependence between the values of different nodes, therefore, the algorithm for determining the PDL of one-stage functional dependences of the parameters of the modes and electric quantities in case of faults to the network according to the PDL of RVs remains fundamentally the same. In the intra-site dependence is taken into account in the following way: the formation of a joint RVs PDF in the work of unconditional PDF within each node to one argument used unconditional PDF, and for another argument conditional PDF another argument provided that the first argument received a specific value in its absolute [17].

In accordance with the meaning of this algorithm, the PDVs of combinations of all RVs are summed up according to the criterion for the equality of each FD with certain values.

These discrete values in the developed algorithm is expedient to form a pre-form a first discrete value as the RV quantiles uniform order in the interval $[0,1]$, which uniquely and securely fixed in the algorithm are converted and controlled.

FD values are generally formed using a single stage of initial data by iterative respectively single and double computing processes [18]: once the process parameters for the modes, two electrical quantities to process in case of faults.

\section{Algorithm}

1. PDL random arguments (active and reactive power load nodes $N_{1}, Q_{1}, \ldots, N_{i}, Q_{i}, \ldots$, active power and voltage of generating units $N_{g 1}, U_{g 1}, \ldots, N_{g k}, U_{g k}, \ldots$ the magnitude and angle of the voltage of the balancing node $U_{b}$ and $\left.\varphi_{b}\right)$ in the absence of a dependents between active and reactive powers in load nodes, active power and voltage in generator nodes:

For the CDFs:

$\left\{\begin{array}{c}f_{1}\left(n_{1}\right), f_{1 q}\left(q_{1}\right), \ldots, f_{i}\left(n_{i}\right), f_{i q}\left(q_{i}\right) \\ f_{g 1}\left(n_{g 1}\right), f_{g 1 u}\left(u_{g 1}\right), \ldots, f_{g k}\left(n_{g k}\right), f_{g k u}\left(u_{g k}\right)\end{array}\right.$

For the PDFs:

$\left\{\begin{array}{c}c_{1}\left(n_{1}\right), c_{1 q}\left(q_{1}\right), \ldots, c_{i}\left(n_{i}\right), c_{i q}\left(q_{i}\right) \\ c_{g 1}\left(n_{g 1}\right), c_{g 1 u}\left(u_{g 1}\right), \ldots, c_{g k}\left(n_{g k}\right), c_{g k u}\left(u_{g k}\right)\end{array}\right.$

For the slack bus, the voltage and angle are taken $U_{b}=$ const and $\varphi_{b}=0$ [19].

Under normal PDL and the correlation between the active and reactive powers of the load nodes, the active power and voltage of the generator nodes, the initial data are the unconditional and conditional CDF and PDF of both components of each node, because during the formation of a joint PDF of all RVs, the unconditional PDF of one from the each node components and the conditional PRV of the other node component are multiplied.

Reasonable preference is usually given as absolute PDL node active power and conditioned PDL gets reactive power in the load node voltage in generator node, i.e.:

For the CDFs:

$$
\left\{\begin{array}{c}
f_{1}\left(n_{1}\right) f_{1 q}^{n_{1}}\left(\frac{q_{1}}{n_{1}}\right), \ldots, f_{i}\left(n_{i}\right) f_{i q}^{n_{i}}\left(\frac{q_{i}}{n_{i}}\right) \\
f_{g 1}\left(n_{g 1}\right) f_{g 1 u}^{n_{g 1}}\left(\frac{u_{g 1}}{n_{g 1}}\right), \ldots, f_{g k}\left(n_{g k}\right) f_{g k u}^{n_{g k}}\left(\frac{q_{g k}}{n_{g k}}\right)
\end{array}\right.
$$

For the PDFs:

$$
\left\{\begin{array}{c}
c_{1}\left(n_{1}\right) c_{1 q}^{n_{1}}\left(\frac{q_{1}}{n_{1}}\right), \ldots, c_{i}\left(n_{i}\right) c_{i q}^{n_{i}}\left(\frac{q_{i}}{n_{i}}\right), \\
c_{g 1}\left(n_{g 1}\right) c_{g 1 u}^{n_{g 1}}\left(\frac{u_{g 1}}{n_{g 1}}\right), \ldots, c_{g k}\left(n_{g k}\right) c_{g k u}^{n_{g k}}\left(\frac{q_{g k}}{n_{g k}}\right)
\end{array}\right.
$$


To form the normal conditional CDF and PDF, it is necessary to specify pair correlation coefficients between the active and reactive powers of each load node $\mathrm{r}\left(N_{1}, Q_{1}\right), \ldots, \mathrm{r}\left(N_{i}, Q_{i}\right), \ldots$ and each generator node $\mathrm{r}\left(N_{g 1}, U_{g 1}\right), \ldots, \mathrm{r}\left(N_{g k}, U_{g k}\right), \ldots$. Also, for this purpose, expected value $(\mathrm{EV})$ are required:

$\left\{\begin{array}{c}\mathrm{m}\left(N_{1}\right), \mathrm{m}\left(Q_{1}\right), \ldots, \mathrm{m}\left(N_{i}\right), \mathrm{m}\left(Q_{i}\right) \\ \mathrm{m}\left(N_{g 1}\right), \mathrm{m}\left(U_{g 1}\right), \ldots, \mathrm{m}\left(N_{g k}\right), \mathrm{m}\left(U_{g k}\right)\end{array}\right.$

and standard deviations (SD):

$\left\{\begin{array}{c}\sigma\left(N_{1}\right), \sigma\left(Q_{1}\right), \ldots, \sigma\left(N_{i}\right), \sigma\left(Q_{i}\right) \\ \sigma\left(N_{g 1}\right), \sigma\left(U_{g 1}\right), \ldots, \sigma\left(N_{g k}\right), \sigma\left(U_{g k}\right)\end{array}\right.$

According to the listed characteristics at normal PDL, EV and SD of the normal conditional PDL are calculated:

$\mathrm{m}\left(Q_{1} / N_{1}\right), \ldots, \mathrm{m}\left(\frac{Q_{i}}{N_{i}}\right), \ldots \mathrm{m}\left({\frac{U}{g_{1}}}_{N}\right), \ldots, \mathrm{m}\left(\frac{U}{N k}_{g k}\right)$

For example, for the $i$-th load node:

$\mathrm{m}\left(Q_{i} / n_{i}\right)=\mathrm{m}\left(Q_{i}\right)+\mathrm{r}\left(N_{i}, Q_{i}\right) \frac{\sigma\left(Q_{i}\right)}{\sigma\left(N_{i}\right)}\left[n_{i}-\mathrm{m}\left(N_{i}\right)\right]$

$\sigma\left(Q_{i} / n_{i}\right)=\sigma\left(Q_{i}\right) \sqrt{1-\mathrm{r}^{2}\left(N_{i}, Q_{i}\right)}$

2. The choice of the quantity and the actual values of the RVs CDF quantile orders [20] from the range $[0,1]$ : $0, p_{1}, \ldots, p_{j}, \ldots, 1$ with a uniform step.

3. Preparation of the RVs basic system [21] quantiles of the same order and the parameters of the PDF components of the regimes: flows of active and reactive power in the branches, values and voltage angles in the nodes is the main task. Electrical values in the event of damage: full currents and their symmetrical components, ratios of residual stresses at the nodes of line connections to currents compensated by a zero sequence in the lead branches of the lines, high-voltage bushings of transformer elements - the second task, which follows from the first, and will be necessary in future work.

The basic system is allocated only in order to build a discrete system of values of all FDs, each in its actual natural range, which are used as enumeration criteria. The solution is deployed for all variants of RV quantiles of the same order for cases where there are no dependencies between pairs of components in nodes and if there is a correlation dependence between these pairs.

If there is no dependency between components in the nodes:

Zero order RV quantiles:

$\left\{\begin{aligned} n_{10} & =f_{1}^{-1}(0), q_{10}=f_{1 q}^{-1}(0) \\ n_{i 0} & =f_{i 1}^{-1}(0), q_{i 0}=f_{1 q}^{-1}(0) \\ n_{g 10} & =f_{g 1}^{-1}(0), \mathrm{u}_{g 10}=f_{g 1 u}^{-1}(0) \\ n_{g k 0} & =f_{g k}^{-1}(0), \mathrm{u}_{g k 0}=f_{g k u}^{-1}(0)\end{aligned}\right.$

Values of RVs PDF from zero order RVs quantiles:

$\left\{\begin{array}{c}c_{1}\left(n_{10}\right), c_{1 q}\left(q_{10}\right), \ldots, c_{i}\left(n_{i 0}\right), c_{i q}\left(q_{i 0}\right) \\ c_{g 1}\left(n_{g 10}\right), c_{g 1 u}\left(u_{g 10}\right), \ldots, c_{g k}\left(n_{g k 0}\right), c_{g k u}\left(u_{g k 0}\right)\end{array}\right.$
The value of the RVs joint PDF from zero order RVs quantiles:

$\left\{\begin{array}{c}c_{1}\left(n_{10}\right) \cdot c_{1 q}\left(q_{10}\right), \ldots, c_{i}\left(n_{i 0}\right) \cdot c_{i q}\left(q_{i 0}\right) \\ c_{g 1}\left(n_{g 10}\right) \cdot c_{g 1 u}\left(u_{g 10}\right), \ldots, c_{g k}\left(n_{g k 0}\right) \cdot c_{g k u}\left(u_{g k 0}\right)\end{array}\right.$

If there is a correlation dependence between the pairs of components in the nodes:

Values of RVs PDF from zero order RVs quantiles:

$\left\{\begin{array}{c}c_{1}\left(n_{10}\right), c_{1 q}^{n 1}\left(q_{10} / n_{10}\right), \ldots, c_{i}\left(n_{i 0}\right), c_{1 q}^{n_{i}}\left(q_{i 0} / n_{i 0}\right), \ldots \\ \mathrm{c}_{g 1}\left(n_{g 10}\right), \mathrm{c}_{g 1 u}^{g 1}\left(u_{g 10} / n_{g 10}\right), \ldots, \mathrm{c}_{g k}\left(n_{g k 0}\right), \mathrm{c}_{g k u}^{g k}\left(u_{g k 0} / n_{g k 0}\right)\end{array}\right.$

The value of the RVs joint Pdf from zero order RVs quantiles:

$\left\{\begin{array}{c}c_{1}\left(n_{10}\right) \cdot c_{1 q}^{n_{1}}\left(q_{10} / n_{10}\right), \ldots, c_{i}\left(n_{i 0}\right) \cdot c_{1 q}^{n_{i}}\left(q_{i 0} / n_{i 0}\right), \ldots \\ \mathrm{c}_{g 1}\left(n_{g 10}\right) \cdot \mathrm{c}_{g 1 u}^{g 1}\left(u_{g 10} / n_{g 10}\right), \ldots, \mathrm{c}_{g k}\left(n_{g k 0}\right) \cdot \mathrm{c}_{g k u}^{g k}\left(u_{g k 0} / n_{g k 0}\right)\end{array}\right.$

A software-computational iterative process is carried out to calculate the parameters of the modes with a variant of the initial data in the form of zeroorder quantiles. As a result, the first FD values are obtained from their ranges as mode parameters such as flows of active and reactive powers at the outer ends of the branches of the longitudinal elements (leading branches of the lines), magnitudes and angles of the node voltage, active and reactive powers, magnitudes and angles of the generator and engine-load currents nodes.

The main goal is to test an improved method for obtaining probabilistic characteristics for tasks of medium RVs dimension, because studies of small-sized FDs have already been successfully carried out [22] and completely coincide with mathematical integration. Accordingly, the study of the parameters of the steady state is of interest, but the loss of power in the lines and the assessment of their CDF and PDF are more interested [23]. The main focus in this study is not the maximum quality of the models used, but the ability to investigate the question with the available tools.

\section{Simulation Model}

The test system used to illustrate probabilistic stability analysis is a 30-Bus IEEE system, which represents a portion of the American Electric Power System as of December 1961. This technical note describes the details of the IEEE 30-bus system [24]. The system, shown in Figure 2, consists of 20 loads, capacitor banks, 41 transmission lines, and 6 conventional generators. 


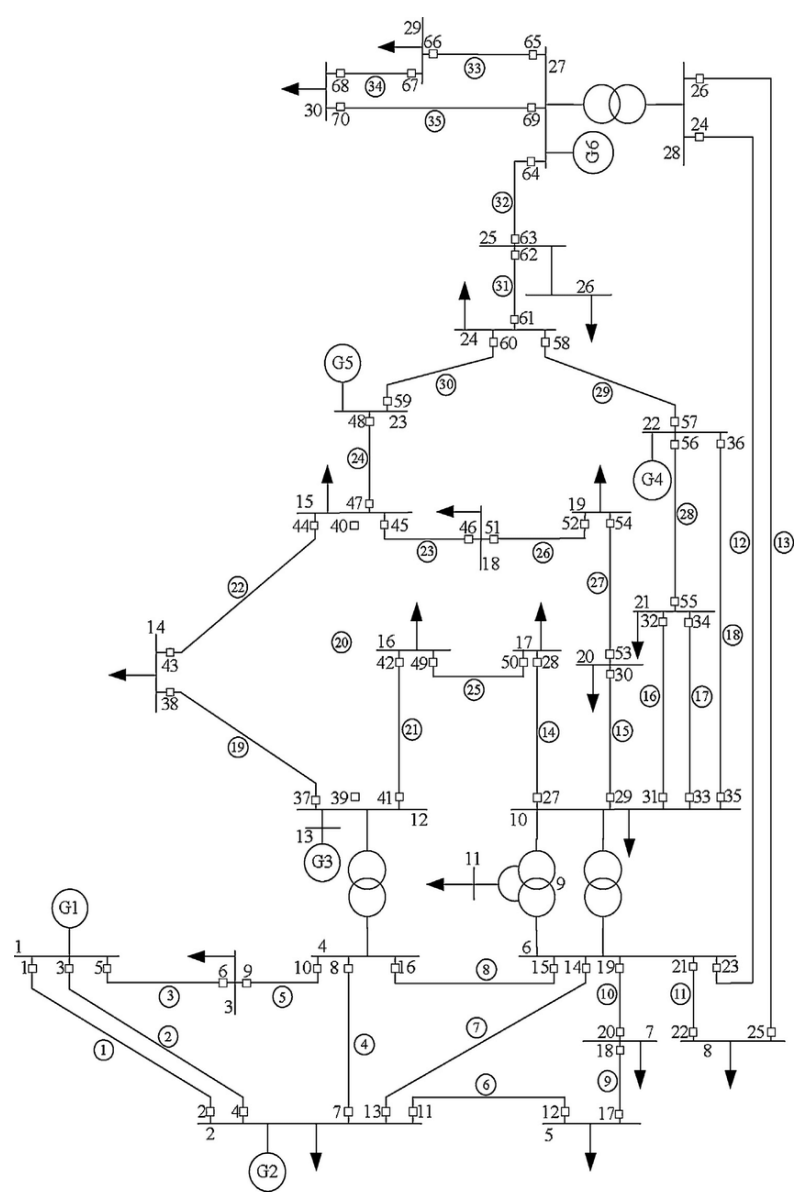

Figure 2. 30-Bus IEEE system

For work, the probability distributions of uncertain system parameters - quantiles and quantile orders of the source data are generated in Matlab. To calculate the power flows, a Matpower (a special package of Matlab ${ }^{\circledR}$ $M$-files) will be used, which is using to calculate power flow (PF) and optimal power flow (OPF) problems [25]. It also has great flexibility in settings and changing input data parameters.

The input data, namely the active and reactive power of loads, active power and voltage on generators, is set according to the algorithm described above. Initially, $m$ and $\sigma$ are defined, and vectors are formed relative to the inverse of the normal CDF with EV and $\mathrm{SD}$, and the PDF of the normal distribution (Table 1).

Table 1. Example of quantiles and PDFs of active power in Generator \#1 node

\begin{tabular}{|c|c|c|c|c|c|}
\hline $\begin{array}{l}\text { Quantile } \\
\text { order }\end{array}$ & $\begin{array}{c}\text { Gen. \#1 } \\
\text { quantiles }\end{array}$ & EV & SD & CDFs & PDFs \\
\hline 1 & 30.9987 & \multirow{6}{*}{48} & \multirow{6}{*}{4.57} & 0.0001 & 0.0086 \\
\hline 2 & 40.0885 & & & 0.0417 & 0.0195 \\
\hline 3 & 41.6802 & & & 0.0834 & 0.0335 \\
\hline 4 & 42.7429 & & & 0.1250 & 0.0450 \\
\hline$\ldots$ & $\ldots$ & & & $\ldots$ & \\
\hline $\mathrm{n}$ & 65.0012 & & & 0.9999 & 0.0086 \\
\hline
\end{tabular}

In the same way, the settings for each RV of the system load and generator nodes are formed. Next, configure the Manpower. In our case, it will be convenient to use in standard mode with ' $A C$ ' - use nonlinear $\mathrm{AC}$ model \& corresponding options and will be solved by the 'NR' method - Newton's method [26]. For convenience of calculation, a special function has been added to the algorithm that uploads data to an external file for unloading RAM, since working with multidimensional arrays can quickly overload the computer. The full algorithm for working with the Matlab program is shown in the Figure 3.

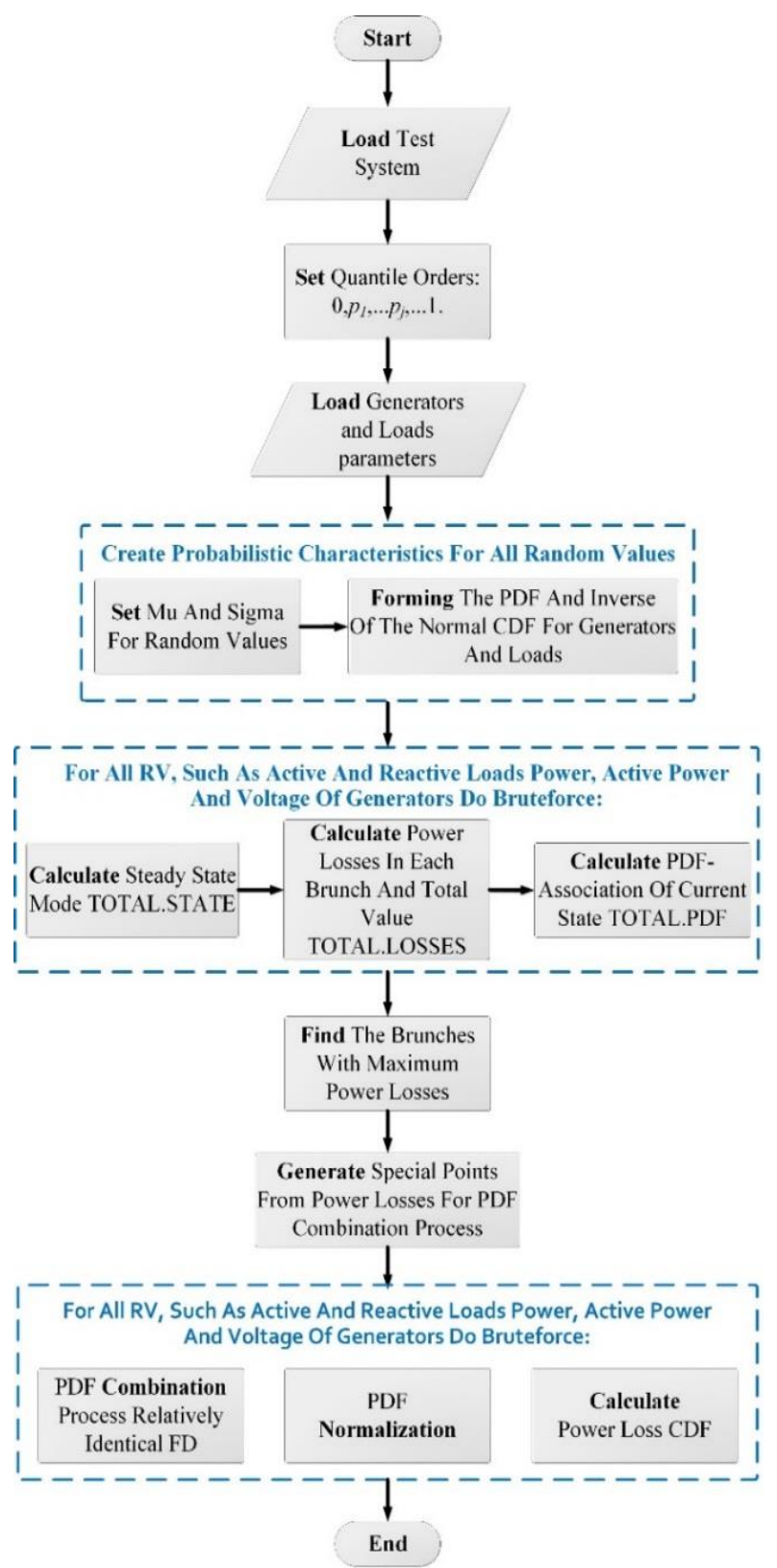

Figure 3. Complete algorithm for calculating the probabilistic characteristics using Matlab Matpower system

\section{Results}

For each iteration of the steady state mode, the function of calculating power losses in the lines is performed. After a complete calculation of all the regime states, the process of calculating the joint PDFs and the calculation and normalization of the final PDFs and CDFs for power losses occur. 
At the end of the process, we can examine the received data. It is worth noting that this procedure was also performed for a system of smaller dimensions (14bus system [27]) in order to compare the nature of PDLs.

In total, power losses in all 41 branches were determined for the 30-bus system, while Figures 4 and 5 represent the results in the apparent power form:

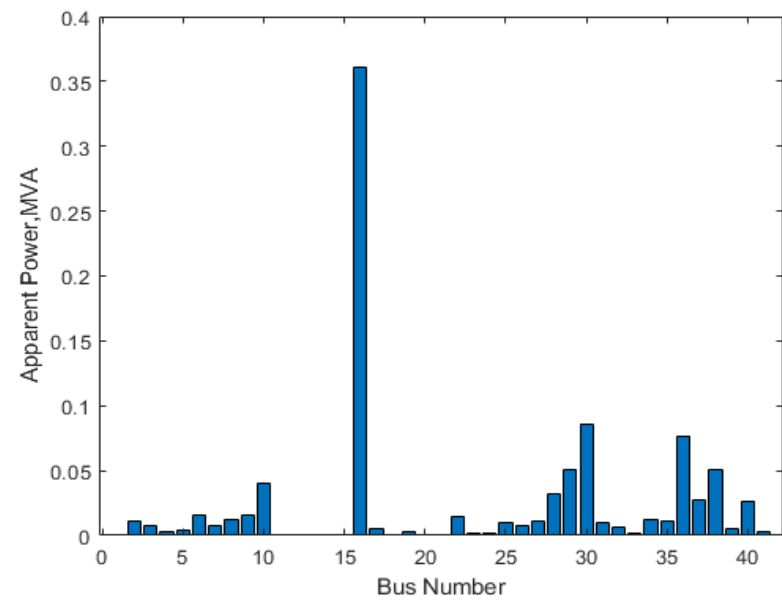

Figure 4. Minimum apparent power losses

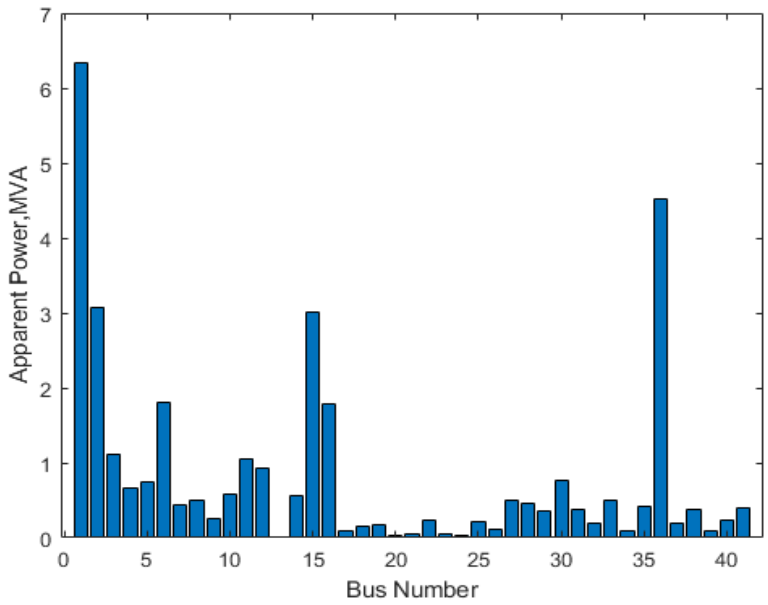

Figure 5. Maximum apparent power losses

The largest losses are observed in lines 1, 2, 15, 36 (Table.2). In other branches, power losses were also observed, however, according to according to Figures 4 and 5 , their influence on the system is extremely insignificant. In this case, the total power loss in the power system can reach 28.31 MVA.

Matpower also has the ability to deterministic calculation of power flow and optimal power flow. In the OPF case, it solves the power flow problem with locational contingency and load-following reserves, ramping costs and constraints. The results are 9.4784 and 13.9047, respectively.
Table 2. Power losses results

\begin{tabular}{l|l|l}
\hline Branch \# & $\begin{array}{l}\text { Minimal Apparent } \\
\text { power loss, MVA }\end{array}$ & $\begin{array}{l}\text { Maximal Apparent } \\
\text { power loss, MVA }\end{array}$ \\
\hline 1 & $1.9838196766536 \mathrm{e}-08$ & 6.33543503428032 \\
\hline 2 & 0.0105102092833261 & 3.07661650239206 \\
\hline 15 & $6.1995800347142 \mathrm{e}-05$ & 3.01102925204714 \\
\hline 36 & 0.0767060899562439 & 4.51128480473431 \\
\hline Total Losses & 2.0805 & 28.3101 \\
\hline PF Losses & 9.4784 & \\
\hline OPF Losses & 13.9047 & \\
\hline
\end{tabular}

However, in our case, more attention should be paid to the probabilistic characteristics of power losses.

In the process of CDF and PDF analysis, namely CDF, because it provides unambiguous FD determination for a given CDF value.

According to the obtained graphs (Figures 6, 7), it is possible to observe the nature of values that are normal or close to the normal distribution law with offset EVs in some cases.

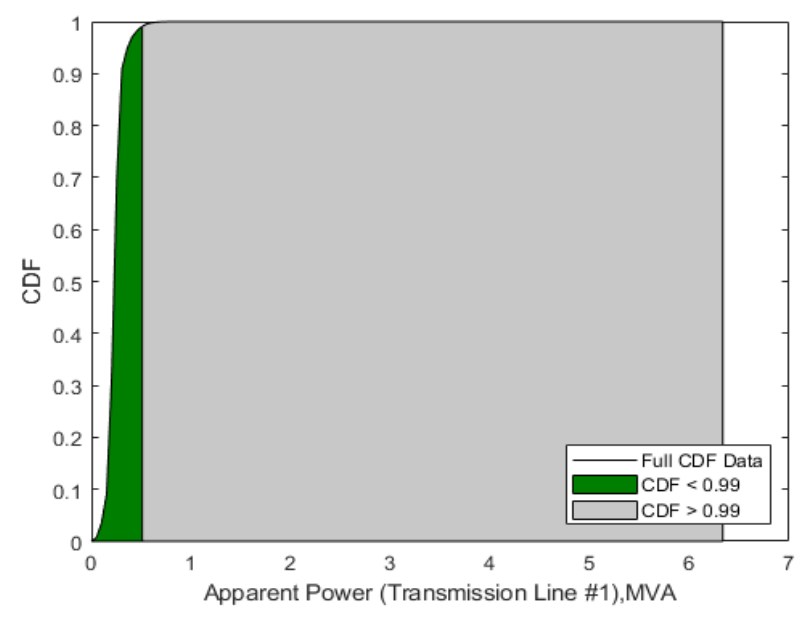

Figure 6. CDF of power losses in transmission line \#1

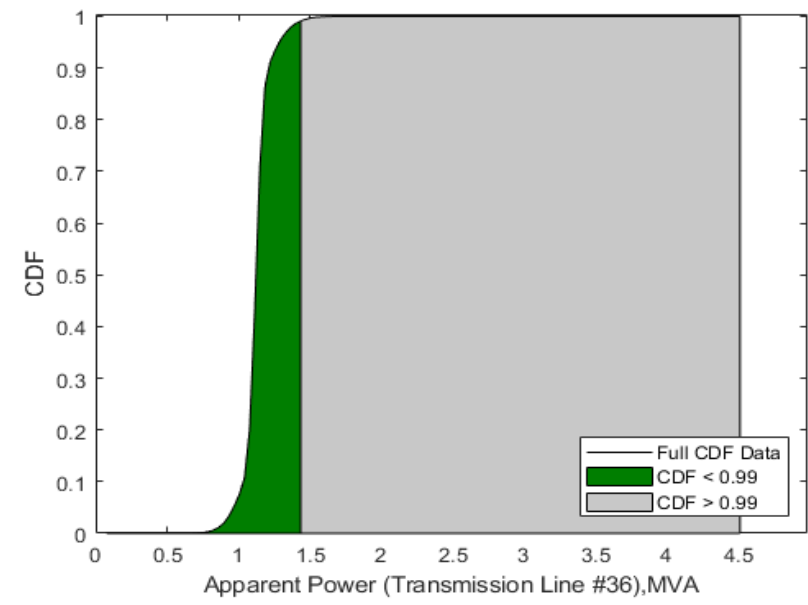

Figure 7. CDF of power losses in transmission line \#36

It can be observed that, for example, on the line №36, the total power loss can reach 4.5112 MVA (in accordance with Table 2). However, due to its distribution parameters, in the case of determining the power value that does not exceed the specified value 
with a $99 \%$ probability (as a margin of error), we have that the power loss value of line with a $99 \%$ probability will not exceed 1.436 MVA, which is only $31.8244 \%$ of the maximum. In a number of other studied lines, the power share that does not exceed $99 \%$ of the probability may be even less - Table 3 does not exceed the FD value law when the situation probability occurring is $99 \%$.

Table 3. Power losses $99 \%$ CDF data

\begin{tabular}{l|l|l|l}
\hline System & Branch \# & $\begin{array}{l}\text { Power losses } \\
\text { (CDF > 0.99), } \\
\text { MVA }\end{array}$ & $\begin{array}{l}\text { Power losses } \\
\text { percentage, \% }\end{array}$ \\
\hline $14 \quad$ bus & 1 & 5.31 & 22.880 \\
\cline { 2 - 4 } system & 2 & 5.16 & 46.8306 \\
\cline { 2 - 4 } & 14 & 6.56 & 62.3002 \\
\hline \multirow{3}{*}{\begin{tabular}{c}
$30 \quad$ bystem \\
\cline { 2 - 4 }
\end{tabular}} & 1 & 0.51 & 8.0654 \\
\cline { 2 - 4 } & 2 & 0.13 & 11.3601 \\
\cline { 2 - 4 } & 15 & 0.34 & 31.8244 \\
\hline & 36 & 1.43 &
\end{tabular}

The faster the CDF increases, the better for power losses analysing and determining the maximum possible losses. However, for the dynamic stability study, this option can have a negative effect, due to the large scatter of possible accepted values in a small FD interval with a minimum CDF. A similar kind of dynamic stability studies by the proposed SIBD method as part of research will be investigated in the following works.

An integral part of the algorithm is to get the power loss PDFs, examples in Figure 8.

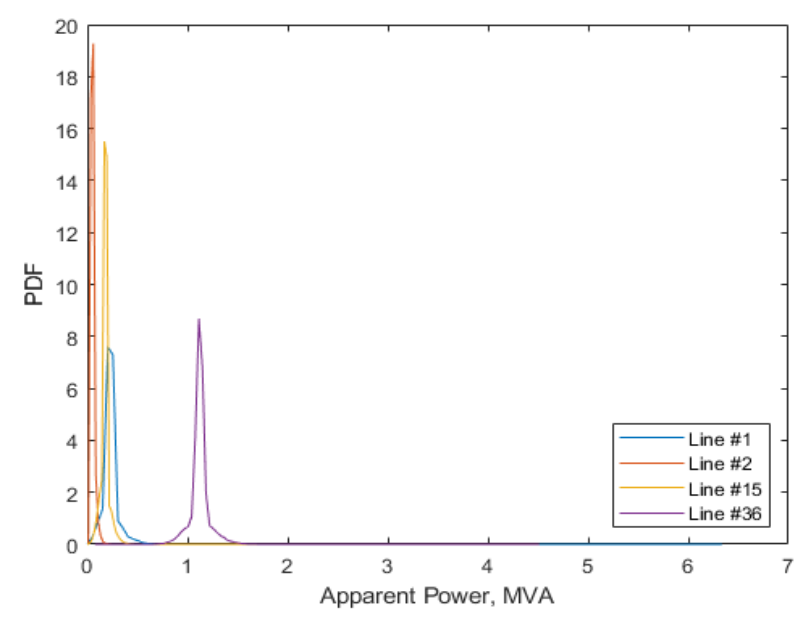

Figure 8. PDF of power losses in the studied lines

Although these curves are not of practical interest in determining the power losses probabilistic characteristics. However, their obtaining determines the possibility of considering the actual and resource value problems, as well as studying the aggregate probabilistic characteristics of RES implementation [28], since they are initially probabilistic in nature - the dependence of power on wind speed, installation location, climatic condition, and the format of combining in wind farm.

\section{Conclusion}

The purpose of this research work was to determine the power losses probability characteristics, since this is one of the important parameters in the further study of dynamic stability and the possible locations determination for RES installing based on the PDLs study.

Due to the special form of input data assignment, in this SIBD method implies the possibility of studying the PDL of the studied quantities in full, and not on the random sampling basis, as is often done on the other methods. Thus, it was possible to avoid solving complex, multidimensional integral problems. Testing of the method on the small-sized FDs has already been carried out in our other studies and is fully consistent with the analytical methods for the RV PDL finding.

The determination of the probabilistic characteristics in general, as well as the steady-state parameters is one of the main tasks in the research of both 'vertical' and 'vertical-horizontal' EPS, especially in connection with a generated power increase and uncertainties in the EPS, respectively.

With the used algorithm for calculating the FD's PDL and the method for calculating the steady-state mode, it was possible to obtain and study the power losses PDLs. Moreover, when studying a number of parameters, it turned out that it is not always necessary to consider maximum power losses, unless it is a matter of setting up a completely stable EPS.

However, since an important factor in the EPS operation is also the amount of expenses, the low cost of the final product for consumers, the use of PDLs even within $99 \%$ of the CDF allows to accurately determine not only the losses probability of a certain FD, but the most important thing is to avoid taking into account the most unlikely losses. Thus, the system is investigated completely in both deterministic and probabilistic form, which allows us to predetermine all its possible states.

The results will be used in the analysis and study of the actual and resource values problem, relative to power losses and the possibility of RES installing, respectively.

\section{Bibliographic References}

[1] VOROPAY, N., Reliability of power supply systems, Novosibirsk: Nauka, 2006.

[2] TRAN-QUOC et al., "Stability analysis for the distribution networks with distributed generation," in: Proceedings of the 2005/2006 IEEE/PES Transmission and Distribution Conference and Exhibition, Dallas, Texas, 2006, pp. 289294.

[3] VENTZEL, E., Theory of probability, Moscow: High school, 1999.

[4] KOPYTOV, E., GRINGLAZ, L., Theory of probability and mathematical statistics, St. Petersburg: Piter, 2004.

[5] GENZ, A., "Numerical computation of rectangular Bivariate and Trivariate normal of $t$ probabilities", Statistics and Computing, 2004, vol. 14, no. 3, pp. 251260, ISSN 0960-3174.

[6] UFA, R., ANDREEV, M., RUBAN, N., SUVOROV, A., GUSEV, A., RAZZHIVIN, I., ASKAROV, A., BAY, Y., KIEVETS, A., LOZINOVA, N., SUSLOVA, O., "The hybrid model of VSC HVDC", Electrical Engineering, 2019, vol. 101, no. 1, pp. 11-18, ISSN 0948-7921. 
[7] MILANOVIĆ, J., "Probabilistic stability analysis: The way forward for stability analysis of sustainable power systems", Philosophical Transactions of the Royal Society A: Mathematical, Physical and Engineering Sciences, 2017, vol. 2100, no 375, pp. 1-22, ISSN 1996-1073.

[8] HUAZHANG, H., CHUNG, C. Y., CHAN, K. W., CHEN, H., "Quasi-Monte Carlo based probabilistic small signal stability analysis for power systems with plug-in electric vehicle and wind power integration", IEEE Transactions on Power Systems, 2013, vol. 28, pp. 3335-3343, ISSN 08858950.

[9] HONG, HP., "An efficient point estimate method for probabilistic analysis", Reliability Engineering \& System Safety, 1998, vol.59, no. 3, pp. 261-267, ISSN 0951-8320.

[10] KARIMISHAD, A., NGUYEN, T. T., "Probabilistic transient stability assessment using two-point estimate method", in: Proceedings of the 8th International Conference on Advances in Power System Control, Operation and Management (APSCOM 2009), Hong Kong, China, 2009, pp. $1-6$.

[11] LI, W., Risk assessment of power systems: models, methods, and applications. Wiley-IEEE Press, 2005.

[12] PRUTIK, A., CHAN, M., SHMOILOV A., "Method of selection of the boundaries of data intervals for determining the laws of distribution of functional dependencies", in: Electricity: from reception and distribution to effective use, Tomsk, Russian, 2010, pp. 1-5.

[13] ANDERSON, P., BOSE, A., "A probabilistic approach to power system stability analysis", IEEE Transactions on Power Apparatus and Systems, 1983, vol. 102, no. 8, pp. 2430-2439, ISSN 0018-9510.

[14] FARIED, S.O., BILLINTON, R., ABORESHAID, S., "Probabilistic evaluation of transient stability of a power system incorporating wind farms", IET Renewable Power Generation, 2010, vol. 4, no. 4, pp. 299-307, ISSN 17521416

[15] HSU, J., Multiple Comparisons: Theory and Methods, London: Chapman and Hall, 1996

[16] NIMMAGADDA, V. N., Probability and Statistical Applications Correlation - Regression, 2014.

[17] JU, P., LI, H., GAN, CH., LIU, Yo., YU, Y., LIU, Yi., "Analytical Assessment for Transient Stability Under Stochastic Continuous Disturbances", IEEE Transactions on Power Systems, 2018, vol. 33, no. 2, pp. 2004-2014, ISSN 0885-8950.

[18] BAY, Y., SHMOILOV, A., ANDREEV, M., SUVOROV, A., KIEVETS, A., RAZZHIVIN, I., "Algorithm of probability distribution law for steady state mode and fault condition parameters of electric power system", Bulletin of South Ural State University. Series "Power Engineering, 2018, vol. 18, no. 4, pp. 13-21, ISSN 1990-8512.

[19] MENGSHOEL, J., CHAVIRA, M., CASCIO, K., POLL, S., DARWICHE, A., UCKUN, S., "Probabilistic Model-Based Diagnosis: An Electrical Power System Case Study", IEEE Transactions on Systems, Man, and Cybernetics - Part A: Systems and Humans, 2010, vol. 40, no. 5, pp. 874-885, ISSN 1083-4427.

[20] CARBONE, P., Schoukens, J., Kollar, I., Moschitta, A., "Measuring the Noise Cumulative Distribution Function Using Quantized Data", IEEE Transactions on Instrumentation and Measurement, 2016, vol. 65, no. 7, pp. 1540-1546, ISSN 0018-9456.

[21] Statios, viewed on 2 April 2020, retrieved form: http://www.statios.com/Resources/03-stats.pdf.

[22] BAY, Y., RAZZHIVIN, I., KIEVETS, A., ASKAROV, A., RUDNIK, V., "Obtaining probabilistic characteristics of electrical quantities and their imbalances" in Electrotehnica, Electronica, Automatica (EEA), 2019, vol. 67, no. 3, pp. 73-80, ISSN 1582-5175

[23] Sultana, U., Khairuddin, A. B., Aman, M.M., Mokhtar, A.S., Zareen, N., "A review of optimum DG placement based on minimization of power losses and voltage stability enhancement of distribution system", Renewable and Sustainable Energy Reviews, 2016, vol. 63 pp. 363-378, ISSN 2302-9285.
[24] Al-roomi, viewed on 10 april 2020, retrieved form: https://al-

roomi.org/multimedia/Power_Flow/30BusSystem/IEEE30B usSystemDATA2.pdf.

[25] Lamaina, P., Sarno, D., Siano, P., Zakariazadeh, A., Romano, R., "A Model for Wind Turbines Placement Within a Distribution Network Acquisition Market", IEEE Transactions on Industrial Informatics, 2015, vol. 11, no. 1, pp. 210-219, ISSN 1551-3203.

[26] Suresh, V., "Comparison of solvers performance for load flow analysis", Transactions on Environment and Electrical Engineering, 2019, vol. 1, pp. 363-378, ISSN 2450-5730.

[27] Al-roomi, viewed on 11 April 2020, retrieved form: https: / /icseg. iti.illinois.edu/ieee-14-bus-system/.

[28] EHSAN, M., AIEN, M., SOROUDI, A., "A probabilistic modelling of photovoltaic modules and wind power generation impact on distribution networks", IEEE Systems Journal, 2012, vol. 6, no. 2, pp. 254-259, ISSN 1932-8184.

\section{Funding Sources}

The work was supported by Ministry of Science and Higher Education of Russian Federation, under the governmental grant "Science", № FSWW-2020-0017.

\section{Authors' Biographies}

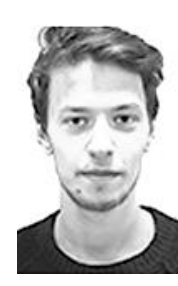

Yuly BAY was born in Tomsk, Russia, in 1991. He received the Dipl.-Ing. in 2014 at Tomsk Polytechnic University. Currently he is an Assistant of Division for Power and Electrical Engineering Tomsk Polytechnic University. He is involved in research work, connected with finding the full probabilistic characteristics of the power industry quantities.

His research interests concern: numerical probabilistic methods and power system simulation.

e-mail address: nodius@tpu.ru

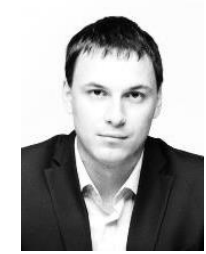

Igor RAZZHIVIN was born in Leninogorsk, Kazakhstan in 1989. He received the M.Sc. in 2015 at Tomsk Polytechnic University. Currently he is an Assistant of Division for Power and Electrical Engineering Tomsk Polytechnic University. He is involved in research work, connected with simulation of renewables.

His research interests concern: relay protection and wind power generation.

e-mail address: lionrash@tpu.ru

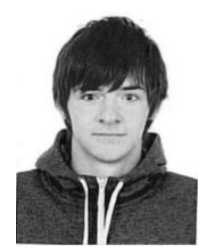

Anton KIEVETS was born in LeninskKuzneckiy, Russia, in 1993. He received the M.Sc. in 2017 at Tomsk Polytechnic University.

Currently he is a Postgraduate of Division for Power and Electrical Engineering, Tomsk Polytechnic University.

His research interests concern: automation control systems and renewables.

e-mail address: kievec.v.l@gmail.com 
Mikhail ANDREEV was born in Sorsk, Russia, in 1987. He received his Engineer and Ph.D. degrees in 2010 and 2013 at Tomsk Polytechnic University. He is currently a Head of R\&D Laboratory for Electrical Power System Simulation and Associate Professor with Division for Power and Electrical Engineering, Tomsk Polytechnic University. $\mathrm{He}$ is involved in research work, connected with relay protection of electric power systems.

His research interests concern: emergency automation of electric power systems.

e-mail address: andreevmv@tpu.ru

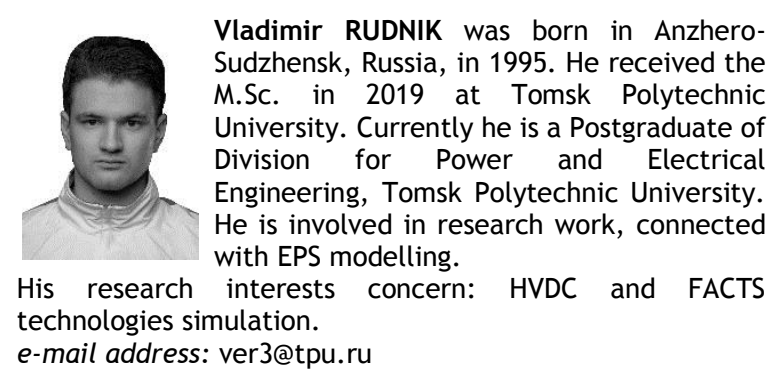

Sudzhensk, Russia, in 1995. He received the

University. Currently he is a Postgraduate of

Division for Power and Electrical

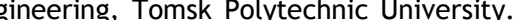

with EPS modelling.

technologies simulation.

e-mail address: ver3@tpu.ru 